\title{
STUDIES OF MICROBIAL POPULATIONS ARTIFICIALLY LOCAL- IZED IN VIVO. I. MULTIPLICATION OF BACTERIA AND DISTRIBUTION OF DRUGS IN AGAR LOCI ${ }^{1}$
}

\author{
By CHARLES A. WERNER, ${ }^{2}$ VERNON KNIGHT, AND WALSH MCDERMOTT WITH \\ THE TECH NICAL ASSISTANCE OF CAROL ADAMS AND REBECKAH DUBOIS \\ (From the Department of Medicine, New York Hospital-Cornell University Medical Center, \\ New York, N. Y.)
}

(Submitted for publication August 5, 1953; accepted January 13, 1954)

Knowledge concerning the action of antimicrobial drugs on susceptible parasites has been gathered almost exclusively from the study of in vitro systems and from animal protection tests. In vitro systems represent a decaying environment which can only approximate for short periods of time and within narrow limits the complex biochemical and physiologic conditions obtaining in vivo. In contrast, within the animal host a continuous supply of nutriments is available to support microbial multiplication for prolonged periods of time, and may well permit greater physiologic heterogeneity among the parasites. In vitro systems do not generally include certain properties of the natural lesion such as necrosis, fibrosis, and parasites located within the cells. Likewise excluded from in vitro systems are certain important drug-host factors which may have a profound effect upon chemotherapeutic action. These include elimination or destruction of drug, binding by plasma proteins, and anatomicophysiologic barriers which may impede passage of drug from one body compartment to another (1).

Studies of the direct action of antimicrobial compounds on bacteria in vivo have been limited by the lack of suitable technics. Animal protection tests have not allowed precise control over the bacterial populations under study. An ideal technic would be one in which the microorganisms could be localized in an animal host in a manner in which the parasites would be subsisting directly upon nutriments supplied by the host but in which

\footnotetext{
1 This study was aided in part by grants from Chas. Pfizer \& Co., Brooklyn, N. Y.; the Lederle Laboratories Division, American Cyanamid Co., Pearl River, N. Y.; and the Division of Research Grants and Fellowships, $\mathrm{Na}$ tional Institutes of Health, U. S. Public Health Service.

2 Postdoctorate Research Fellow, National Institutes of Health, U. S. Public Health Service during the period in which this study was conducted.
}

they would be available for removal for quantitative studies at desired intervals. Only a few attempts have been made to develop such technics. Eagle, Fleischman, and Musselman (2) have injected pneumococci and streptococci directly into the thigh muscle of the mouse and the testicle of the rabbit. With this method no attempt is made to ensure localization of the bacteria. Lurie inoculated phagocyted tubercle bacilli into the anterior chamber of the eye of the rabbit in order to study the responses of immune and non-immune animals (3). The same investigator has also injected animals subcutaneously with suspensions of virulent tubercle bacilli in melted agar and has implanted small collodion bags containing tubercle bacilli suspended in agar in the peritoneal cavities of rabbits (4). These agar inocula were well penetrated by the tissue fluids and supported growth of the tubercle bacilli in vivo.

In the present study the suitability of unenriched agar as a medium for isolating microorganisms growing in vivo was investigated further. A technic was devised whereby pathogenic bacteria were incorporated into the central portion of triple layered agar discs which were inserted into the peritoneal cavities of experimental animals for varying periods of time. The discs containing bacteria could be conveniently removed for quantitative study at appropriate intervals. In addition, the transfer of penicillin, streptomycin, chlortetracycline, and chloramphenicol across the agar to the parasites has been determined. A description of this technic which was originally suggested by the work of Frost (5), and the results obtained, constitute the basis of the present report.

\section{MATERIALS AND METHODS}

Preparation of agar. A 3 per cent suspension of fine granular agar (Difco Bactoagar) in distilled water, $\mathrm{pH}$ 6.0 , in Erlenmeyer flasks was autoclaved at $250^{\circ} \mathrm{F}$. for 30 


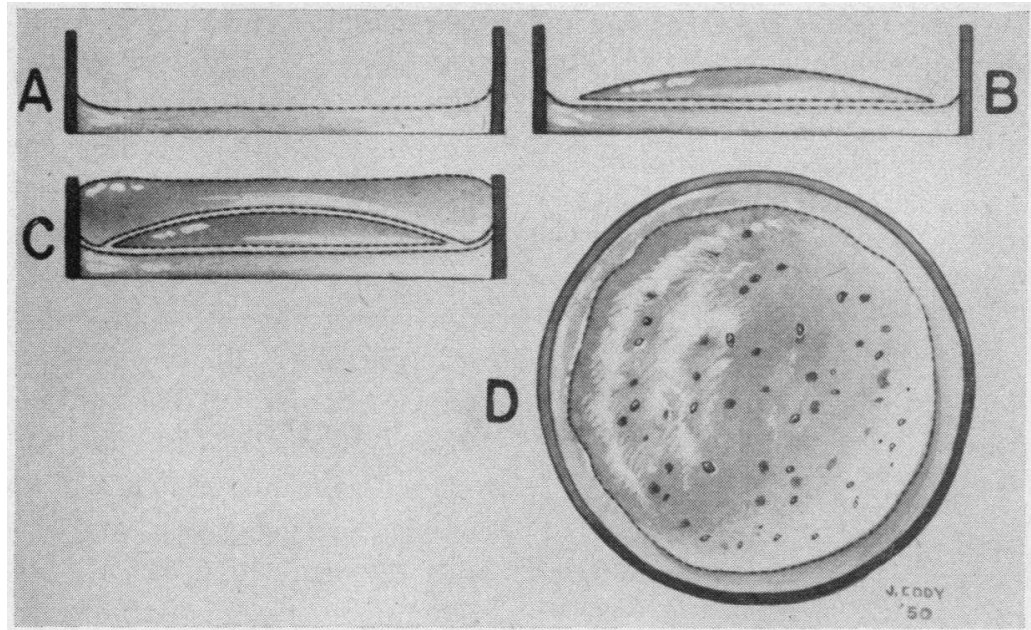

Fig. 1. Steps in the Construction of a Triple Layered Agar Disc

$\mathrm{A}, \mathrm{B}, \mathrm{C}$. Cross sections of the disc showing the relationship of the three layers in the aluminum cylinder. D. Top view of a completed disc containing bacterial colonies.

minutes. The flasks were stored at $4^{\circ} \mathrm{C}$. after the agar had solidified. At the time of an experiment, a flask of agar was placed in boiling water, and the melted agar was then maintained at the desired temperature in a water bath. In addition to the unenriched agar in distilled water, a small quantity of 3 per cent agar was prepared in buffered phosphate solution, $\mathrm{pH}$ 7.6. Ordinary 1.5 per cent beef heart infusion agar with or without added human serum or rabbit's blood was also used in certain experiments.

Preparation of agar discs. Agar was poured under sterile precautions into clean aluminum cylinders $7 \mathrm{~mm}$. high and one $\mathrm{mm}$. thick with an inside diameter of $22 \mathrm{~mm}$. which were set in petri dishes resting on a cold surface. Triple layered agar discs containing inocula of bacteria were prepared as shown in Figures 1 and 2. The bottom layer consisted of 2 to $2.5 \mathrm{ml}$. of melted agar which filled the lower one-third to one-half of the cylinder and sealed the disc at its perimeter. Preliminary dilutions of culture were carried out in 0.85 per cent sterile saline, and a final dilution of $10^{\circ}$ or $10^{7}$ was made in agar at 45 to $50^{\circ} \mathrm{C}$. in a water bath so that essentially no nutritive material was transferred. The inoculated agar was thoroughly mixed, and approximately $0.3 \mathrm{ml}$. was dcposited from the tip of a warmed $2 \mathrm{ml}$. pipet upon the center of the hardened bottom layer. The agar of this central layer was led to within 2 to $2.5 \mathrm{~mm}$. of the inner wall of the cylinder and did not come in contact with the cylinder. A final layer of 2 to $2.5 \mathrm{ml}$. of sterile agar was poured over the previous layers to fill the remaining volume of the cylinder.

In the completed disc, therefore, the central inoculated layer was completely sealed by sterile agar approximately 2 to $3 \mathrm{~mm}$. thick. The whole procedure was accomplished as quickly as possible in order to minimize exposure of the microorganisms to the heat. The triple layered agar discs were used to study the growth of pathogenic bacteria in various animal hosts and in vitro in various media.

Single layered agar discs were prepared by delivering 4 or $5 \mathrm{ml}$. of agar into an aluminum cylinder and allowing the agar to solidify. These discs were used in studying the passage of drugs into agar in vitro and in vivo.

\section{OBSERVATIONS}

\section{Bacterial growth in agar discs}

The ability of unenriched 3 per cent agar to support bacterial growth was tested under a variety of conditions.

The following strains of bacteria were employed: Diplococcus pneumoniae, Type I; Streptococcus pyogenes (C203); Streptococcus zymogenes; Staphylococcus au-

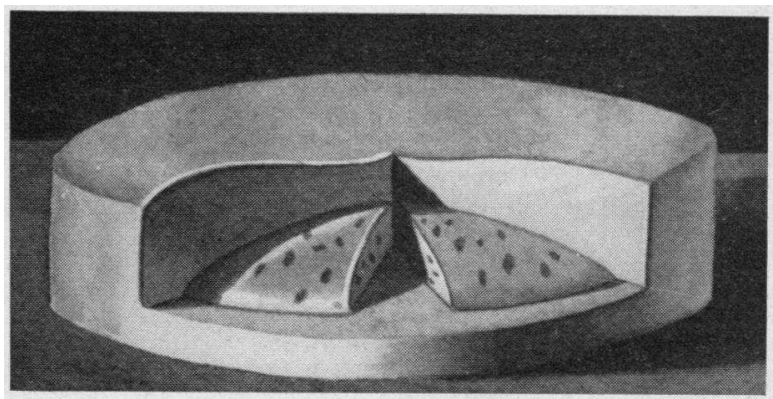

Fig. 2. Diagrammatic Representation of a Completed Disc Containing Bacterial Colonies

The metal ring has been removed and the disc has been cut open to show the internal construction. 
reus; Klebsiella pneumoniae, Type A-D; Salmonella typhosa; Brucella melitensis; Bacillus anthracis; and $M y$ cobacterium tuberculosis ( $\mathrm{H} 37 \mathrm{Rv})$. Cultures were incubated 18 or 24 hours at $37^{\circ} \mathrm{C}$. in infusion broth or 2 per cent blood broth prior to use, except that $\mathrm{Br}$. melitensis culture was a 96-hour growth in trypticase soy broth and $M$. tuberculosis culture was a 96-hour growth in liquid oleic acid-albumin medium.

Bacterial density and homogeneity of the cultures were determined by conventional methods at the start of each experiment, and bacterial growth was studied as follows:

(a) Cultures streaked on the surface of buffered and unbuffered agar, and pour plates of $10^{6}$ dilutions of culture in sterile saline were sealed against drying and were incubated at $37^{\circ} \mathrm{C}$. for 48 hours to 5 days.

(b) Triple layered agar discs in which the central layer consisted of approximately $0.3 \mathrm{ml}$. of agar comprising a $10^{6}$ or $10^{7}$ dilution of culture were incubated at $37^{\circ} \mathrm{C}$. for 24 to 48 hours in deep petri dishes flooded with 60 to $100 \mathrm{ml}$. sterile saline and with infusion broth or 5 per cent serum broth, respectively. Discs inoculated with $\mathrm{Br}$. melitensis were incubated in trypticase soy broth for periods up to five days.

(c) Triple layered agar discs similarly prepared were inserted into the peritoneal cavities of rabbits, guinea pigs and cats. The animals were anesthetized with ether, chloroform, or intravenous pentobarbital, and the peritoneal cavity was opened through an abdominal incision using aseptic surgical technic. The discs with their surrounding aluminum rings were directed into various parts of the peritoneal cavity in a manner to ensure maximum contact with peritoneal surfaces. The wounds were closed, and the animals were permitted to recover consciousness. The discs were removed through the same or a separate incision 24 hours later for examination. If no bacterial colonies were observed the discs were reinserted for an additional 24-hour period or longer. Discs which were found to contain colonies were transferred to sterile petri dishes and were opened with a sterile spatula. The organisms within the discs were subcultured on appropriate media, and their identity was confirmed by study of morphology, gram stain, and agglutination in appropriate antiserum. Peritoneal cultures were made at the times of insertion and removal of the agar discs in order to determine whether contamination of the peritoneal cavity had occurred during the procedure.

Unenriched agar. Agar without added nutrient permitted only minimal growth of a few strains of bacteria. When cultures were streaked on the surface of 3 per cent agar plates only a few, very tiny and irregular colonies barely visible to the unaided eye were apparent after 24 hours of incubation for staphylococci, enterococci, and $S$. typhosa. Somewhat larger colonies of $\mathrm{Kl}$. pneu- moniae and B. anthracis were noted. No discernible colonies of C203 streptococci, type I pneumococci, or $\mathrm{Br}$. melitensis were detected on microscopic examination of the plates even after five days of incubation. No difference was noted with agar which had been buffered to $\mathrm{pH}$ 7.6. In the pour plates and in the triple layered agar discs which were incubated in saline, Kl. pneumoniae was the only microorganism which regularly formed colonies. At 24 hours these colonies were 100 to 150 microns in size and could be detected only with the microscope, while at 48 hours minute colonies could be seen in the depths of the agar with the unaided eye. $M$. tuberculosis was not tested under these conditions.

Enriched agar. In contrast to the meager growth in the unenriched agar, colony development was luxuriant and regular in triple layered agar discs incubated in broth or 5 per cent serum. Numerous large colonies were noted for all species and were comparable to colonies appearing in conventional pour plates of highly nutritive agar. Colonies of $\mathrm{Br}$. melitensis were first visible at 72 hours while those of the other species were apparent at 12 to 24 hours. The tubercle bacillus was not tested.

Agar discs in vivo. Three to five triple layered agar discs inoculated with bacteria were implanted intraperitoneally in each of two rabbits, guinea pigs, and cats, respectively, for each of the aforementioned organisms except that the tubercle bacillus was studied only in rabbits. $\mathrm{Kl}$. pneumoniae, S. typhosa, and B. anthracis consistently grew numerous large colonies in the depths of the discs within 24 hours in all animals (Figure 3 ). Growth of the gram positive cocci and $\mathrm{Br}$. melitensis was less regular. The staphylococcus formed numerous visible but very small colonies (Figure 4). The C203 streptococci, type I pneumococcus, and enterococcus regularly formed microscopic colonies 50 to 100 microns in size within 24 to 48 hours in all discs and a small to moderate number of larger colonies visible to the unaided eye in the majority of discs. $\mathrm{Br}$. melitensis formed many microscopic colonies, 30 to 50 microns, in the depths of the discs in 72 hours, but the colonies did not attain visible size even after five days of incubation. No colonies of $M$. tuberculosis were noted in discs which had remained in rabbits for as long as 20 days. In retrospect, however, the 
conditions of the experiment were of a sort known to be unfavorable for the growth of human tubercle bacilli.

Contamination of the peritoneal cavity by the organism which had been incorporated in the agar occurred in about 25 per cent of trials. This was most frequently encountered with $\mathrm{Kl}$. pneumoniae and $S$. typhosa and occurred more often in guinea pigs than in rabbits or cats. In addition, contamination of the peritoneal cavity with $B$. proteus sometimes occurred. Occasionally peritoneal cultures were found to be positive for the organism under study immediately after a number of discs had been inserted and before bacterial multiplication had begun.

\section{Peritoneal reaction to implanted discs}

The tissue reaction to the agar discs was carefully noted at the time of removal from the animals. In addition, discs which had been inoculated with $M$. tuberculosis and which had been implanted in

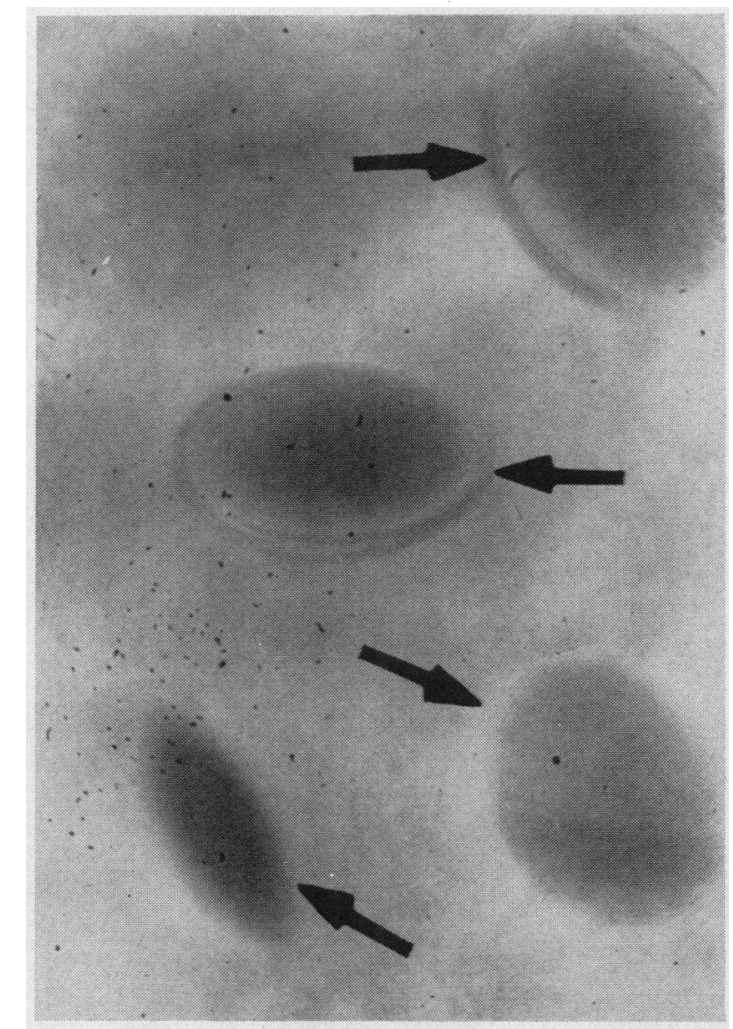

Fig. 3. Four Colonies of Salmonella typhosa (INdicated by Arrows) in the Depths of an Agar Disc Removed from the Peritoneal Cavity of a Guinea Pig After 24 Hours of Incubation $(110 \times)$

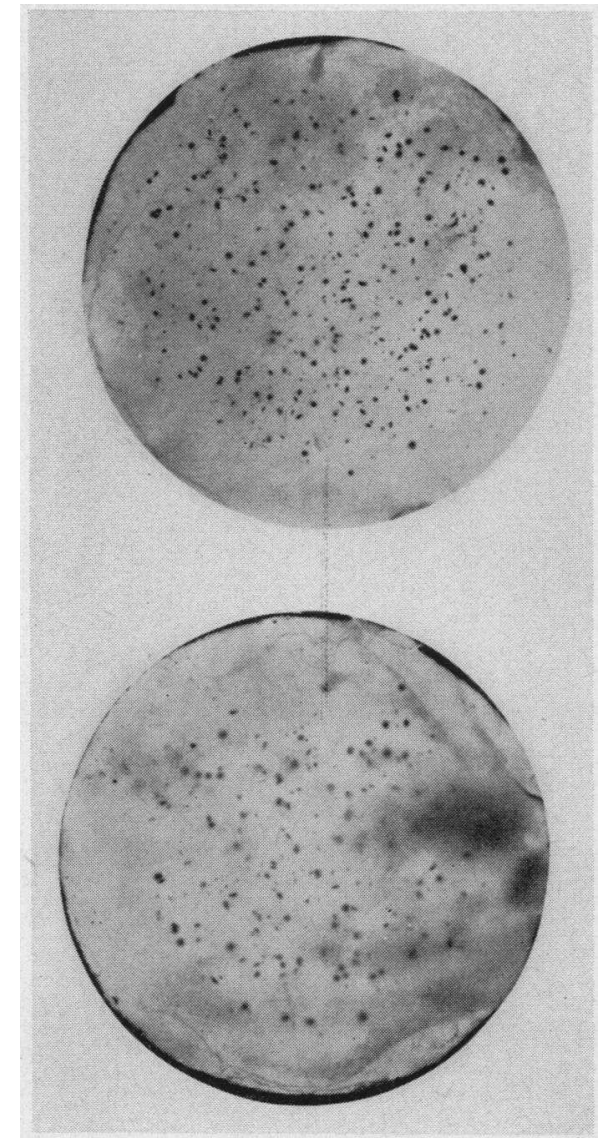

Fig. 4. Triple Layered Agar Discs Inoculated with Staphylococcus aureus and Incubated 24 Hours in the Peritoneal. Cavity of a Guinea Pig, Showing Colony Formation in the Depths of the Disc

rabbits for periods of one to 20 days were fixed in Bouin's solution immediately on removal. These were sectioned perpendicularly to the flat surface and were stained with hematoxylin and eosin, Giemsa's stain, and carbol fuchsin stain for microscopic examination.

A conspicuous tissue reaction to the agar discs implanted in rabbits occurred not only with discs inoculated with bacteria but also with plain agar discs. Within 24 hours the discs were completely enveloped in a thin fibrinous film which increased in thickness to a millimeter or more by 48 to 72 hours. The film of exudate could be easily stripped away from the agar, revealing the smooth surface of the disc.

Microscopic examination of sections of agar discs inoculated with tubercle bacilli and removed from rabbits revealed the exudate to consist pre- 
dominantly of fibrin and polymorphonuclear leukocytes with a smaller proportion of round cells at 24 hours. After 48 hours increasing numbers of mononuclear cells and fibroblasts were noted. Although inflammatory cells were present in large numbers in the exudate on the surface of the agar and had infiltrated the agar along small cracks in the surface or along the interfaces between the different layers, the cells were not able to penetrate the substance of the agar (Figure 5). After seven to ten days small bits of agar had become broken off the surface of the discs and were completely surrounded by organizing exudate.

The tissue reaction in guinea pigs was of the same type as that of the rabbits, but it was less intense and the exudate was not so thick. In cats, on the other hand, little or no exuclate was found surrounding the discs, but the discs were often wrapped in hyperemic omentum. In rabbits and guinea pigs the omentum less frequently enveloped the discs in the peritoneal cavity.

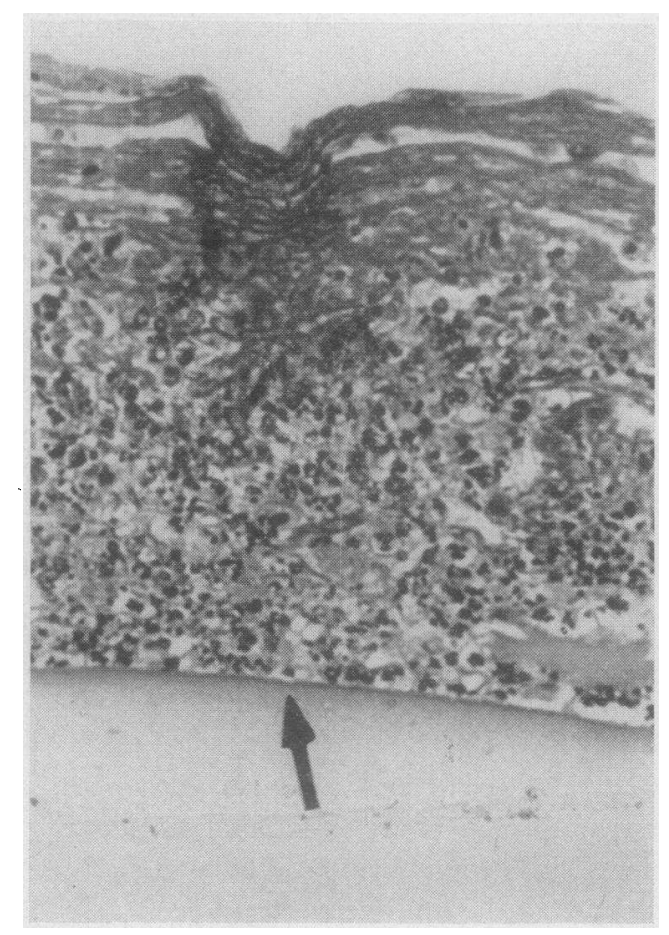

Fig. 5. Section through an Agar Disc Removed from the Peritoneal Cavity of a Rabit after 48 Hours of Incubation Showing the Nature of the Exudate upon the Surface of the Disc

The cells have not penetrated the surface of the agar (indicated by arrow). Hematoxylin and eosin stain $(430 \times)$

\section{Multiplication of S. typhosa in agar discs in the cat}

Triple layered discs were prepared which contained exactly $0.3 \mathrm{ml}$. of agar consisting of a $10^{7}$ dilution of 18 hour culture of $S$. typhosa of known bacterial density. Seven discs were introduced into the peritoneal cavity of a cat anesthetized with intravenous sodium pentobarbital and the edges of the laparotomy wound were drawn together with temporary sutures which could be opened and closed at will. One disc was removed from the animal at $0,1 / 2,1,2,3$, and 4 hours, respectively, while the seventh disc remained inside for 24 hours. On removal each disc was transferred to a sterile mortar containing a small amount of sterile sand in $6 \mathrm{ml}$. of sterile saline and was ground under sterile precautions for 10 minutes. An aliquot was then removed and appropriate dilutions were made in saline. Infusion agar pour plates of these dilutions were incubated for 24 hours and the total numbers of bacilli present in the discs were computed.

Three discs similarly prepared were incubated in zitro in infusion broth for 24 hours. The number of colonies present in these three discs and in the disc which had remained in the peritoneal cavity for 24 hours yielded the average number of bacilli per disc at the start of the experiment. These discs further served as controls on the possible destructive effect on the bacteria of the grinding with sand. Control discs were similarly ground and plated after four hours of incubation in vitro in the dry state and in petri dishes flooded with saline, respectively. These discs served to control possible growth of typhoid bacilli in unenriched agar.

Growth curves of $S$. typhosa in cats have been shown in Figure 6. The microorganisms promptly entered the logarithmic growth phase within four hours in the intraperitoneal discs while no significant multiplication occurred in the control discs.

\section{Penetration of drugs in 3 per cent agar}

Penetration in vitro. The rate of diffusion of penicillin, ${ }^{3}$ streptomycin, ${ }^{4}$ chlortetracycline ${ }^{4}$ and chlorampheni$\mathrm{col}^{5}$ between 3 per cent agar and broth was studied under two sets of circumstances:

(a) Five $\mathrm{ml}$. quantities of broth containing a known concentration of drug were exposed to single layered agar discs of equal volume which had been finely diced with a sterile spatula:

(b) Single layered $5 \mathrm{ml}$. agar discs containing a known concentration of drug were finely diced and were exposed to equal volumes of plain broth.

\footnotetext{
3 Supplied as crystalline penicillin $\mathrm{G}$ and as streptomycin sulfate by Chas. Pfizer \& Co., Brooklyn, N. Y.

4 Supplied as Aureomycin Hydrochloride by the Lederle Laboratories Division, American Cyanamid Co., Pearl River, N. Y.

5 Supplied as Chloromycetin ${ }^{\circledR}$ by Parke, Davis and Co., Detroit, Mich.
} 


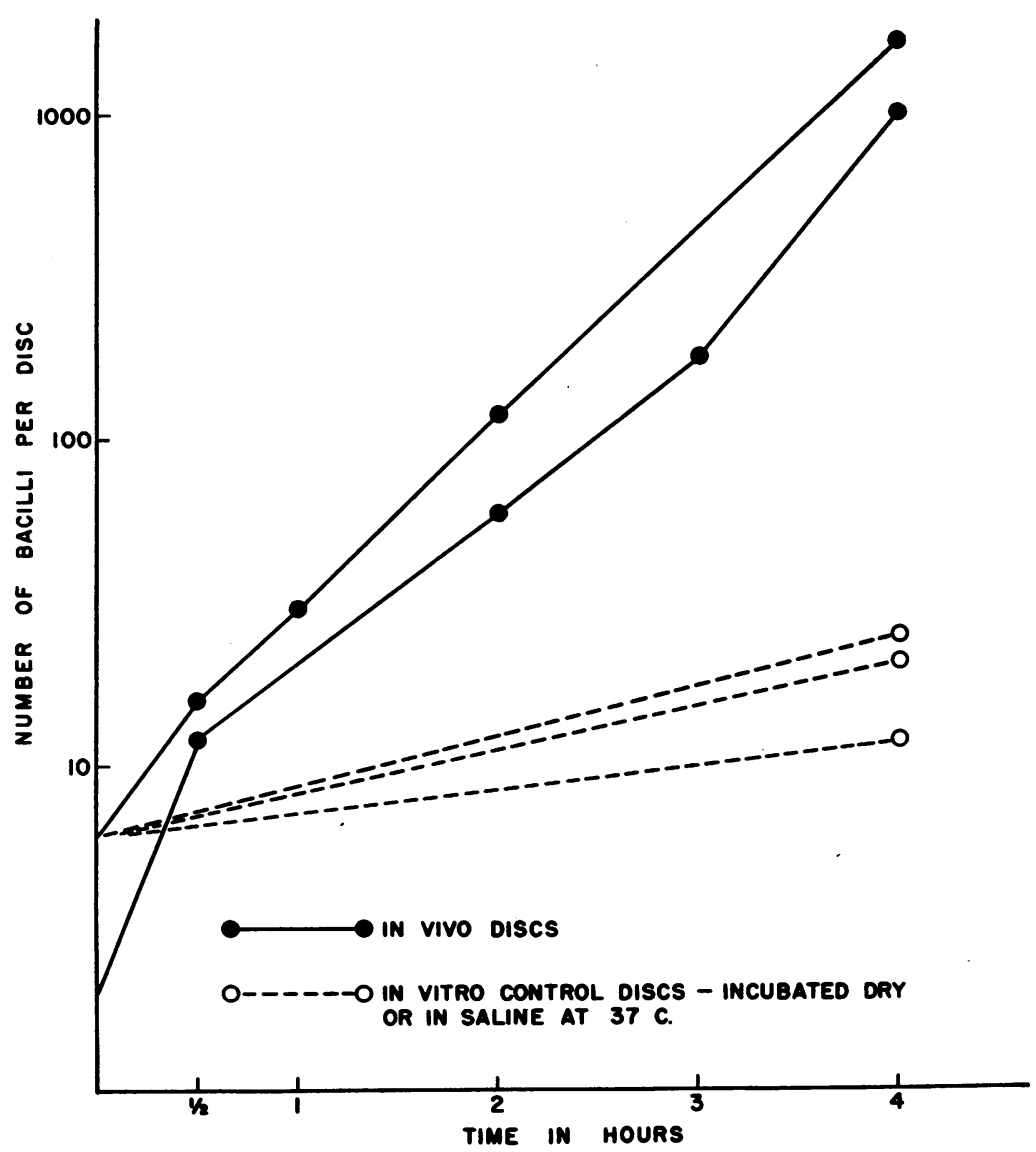

Fig. 6. Rate of Growth of S. typhosa in Agar Disc in Peritoneal Cavity OF A CAT

Two series of five large test tubes containing these respective combinations of broth, agar, and drug were incubated at $37^{\circ} \mathrm{C}$. after vigorous shaking. The broth was removed by pipet as completely as possible from one tube in each series at 1/2, 1, 2, and 3 hours, respectively, for assay of drug concentration. The initial concentration of drug in broth and in agar at the start of each experiment was 20 micrograms per $\mathrm{ml}$. for penicillin, 50 micrograms per $\mathrm{ml}$. for streptomycin, and 200 micrograms per $\mathrm{ml}$. for chlortetracycline and chloramphenicol.

To control the effect which heat may have in reducing the antibacterial potency of these compounds, broth which contained drug was exposed for comparable periods of time in a water bath to temperatures equivalent to those to which the drug was exposed in the melted agar. In addition a standard solution of drug in broth was incubated concurrently with the two series of tubes for each compound. Aliquots of the standard were removed at the same intervals. All specimens of broth were immediately frozen and were stored at $-18^{\circ} \mathrm{C}$. Microbiologic assay was carried out by means of a fractional dilution technic capable of detecting smaller changes in drug con- centrations than the conventional twofold serial dilution method (6).

Three to six determinations were made for each drug in order to control such variables as the degree of dicing of the agar and agitation of the tubes. The results have been recorded in Table I and in Figure 7 . When diced 3 per cent agar discs were added to an equal volume of broth containing penicillin, streptomycin, chlortetracycline, or chloramphenicol, the concentration of drug in the broth fell progressively, indicating the transfer of these drugs into the agar. Allowing for deterioration of drug due to heat as indicated by loss of potency of the standard, the concentration of drug in the broth fell within 30 to 60 minutes to a value approximately one-half the initial concentration or one-half the concentration of the standard after a comparable period of incubation. Conversely when diced agar discs containing known concentra- 
TABLE I

Rate of diffusion of penicillin, streptomycin, chlortetracycline and chloramphenicol between 3 per cent agar and brcth at $37^{\circ} \mathrm{C}$.

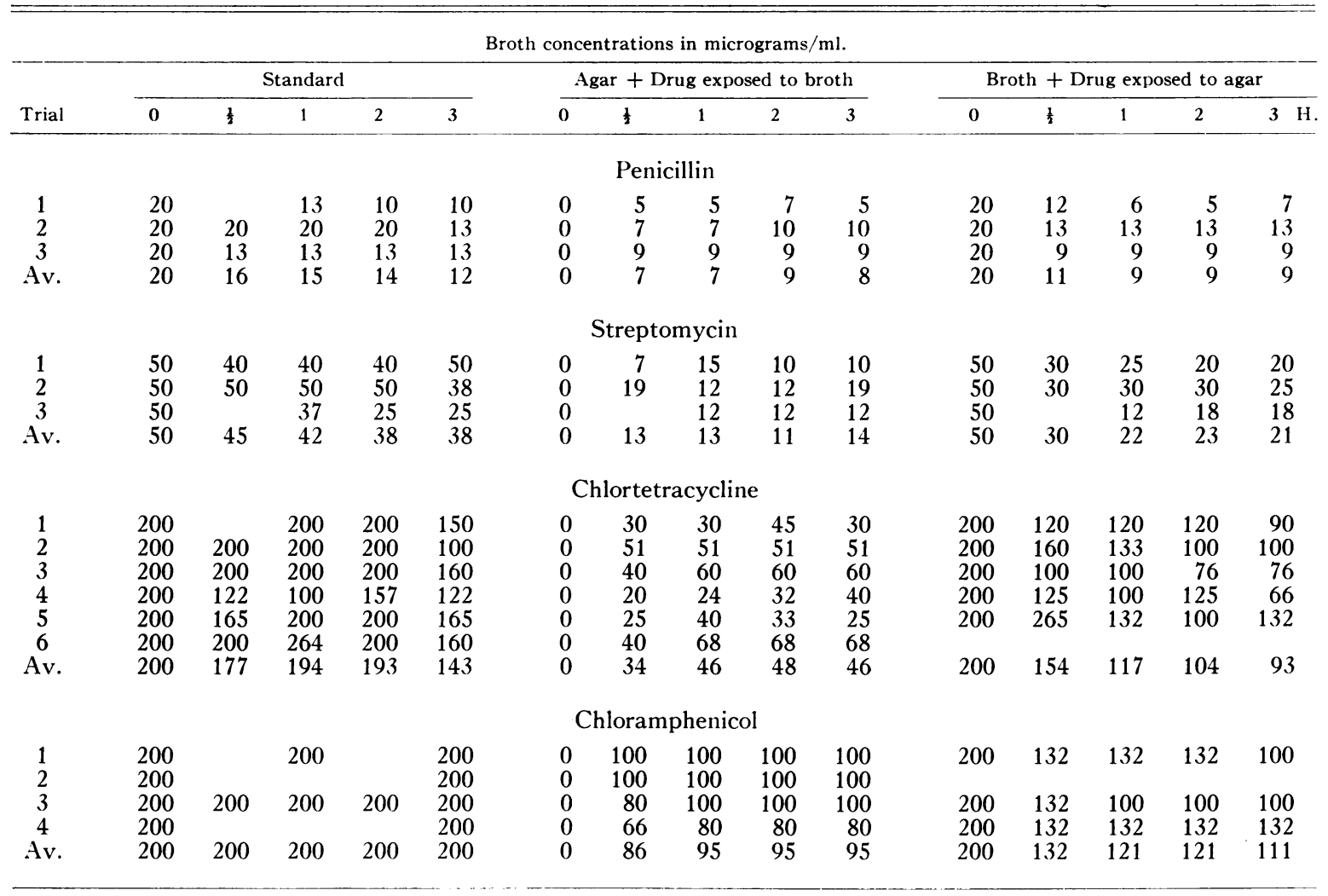

tions of these four drugs were added to equal volumes of plain broth, the concentration of drug in the broth rose progressively. Within a period of 30 to 60 minutes the drug in the broth approached a concentration approximately one-half the initial concentration of drug in agar or one-half the concentration of the standard after a comparable period of incubation.

In the case of penicillin and chloramphenicol, almost exactly equal distribution of drug between agar and broth was achieved in this in vitro system. For streptomycin and chlortetracycline, however, the concentrations in the broth which had been exposed to agar containing drug were only 50 per cent of those which would have obtained had equal distribution of these two drugs between agar and broth occurred. This yielded a factor of two for penicillin and chloramphenicol and a factor of four for streptomycin and chlortetracycline, respectively, in determining the original concentration of these drugs in the agar by this method.
Differences in the values in Table I for successive determinations generally corresponded to a difference of only one or two dilutions in the fractional dilution assay which is within the limits of error of this test. These results validated the equilibration method of estimating the drug content of the agar discs.

Penetration in sico. Rabbits or cats were weighed and were anesthetized with intravenous sodium pentobarbital. Six to eight $5 \mathrm{ml}$. agar discs were introduced into the peritoneal cavity with care to ensure contact of the flat surfaces of the agar discs with the peritoneum and to prevent apposition of discs with each other. After all discs had been inserted the animals received a large intravenous dose of penicillin, streptomycin, or chlortetracycline or a large oral dose of chloramphenicol. Specimens of blood were obtained from the exposed femoral artery or by intracardiac puncture and agar discs were removed $1,2,4,6,8,24$, and 48 hours after drug administration. In animals receiving drug by the intravenous route, additional specimens were collected at 5,15 , and 30 minutes. The blood was allowed to clot for one hour at $4^{\circ} \mathrm{C}$. and the serum was removed after centrifugation. 
The discs were diced with a sterile spatula and were transferred to large test tubes containing an equal volume of broth. After vigorous shaking the tubes were incubated at $37^{\circ} \mathrm{C}$. for one hour to permit equilibration of drug. All specimens of serum and broth were frozen and were stored at $-18^{\circ} \mathrm{C}$. until time of assay. Microbiologic assay was carried out by means of the conventional twofold serial dilution technic, and the concentration of drug in the agar discs was taken to be twice that obtained in the broth for penicillin and chloramphenicol and four times for streptomycin and chlortetracycline. Standard solutions of drug in broth were assayed concurrently with the unknown specimens.

After the six or eight-hour specimens had been obtained the wounds were closed and the animals were allowed to regain consciousness. The animals were reanesthetized and new incisions were made at 24 and 48 hours. In order to prevent dehydration, the animals received by gavage $50 \mathrm{ml}$. of 10 per cent glucose in one per cent saline at 4 and 24 hours after the start of the experiment.

The rate of penetration of agar discs within the peritoneal cavity by penicillin, streptomycin, chlortetracycline, and chloramphenicol and the relation of the serum concentrations to the concentrations in agar have been shown in Figure 8. High concentrations were readily attained in the agar discs. After intravenous administration to rabbits, penicillin and streptomycin rapidly accumulated in the agar discs, and concentrations equal to or exceeding the corresponding serum concentrations were achieved between one and two hours for penicillin and between one and four hours for streptomycin, respectively. In cats given chlortetracycline intravenously, measurable amounts of drug were present in agar discs removed from the peritoneal cavity 30 minutes after injection. The concentrations rose more slowly than those of penicillin and streptomycin, however, and did not attain the same magnitude as the serum concentrations. Measurable concentrations of chloramphenicol were present in discs removed from cats one or two hours after oral administration of this drug but remained below those in the serum. Concentrations of drug in the agar discs fell slowly, and measurable concentrations of all drugs except penicillin were still present 24 hours after administration of the single doses.
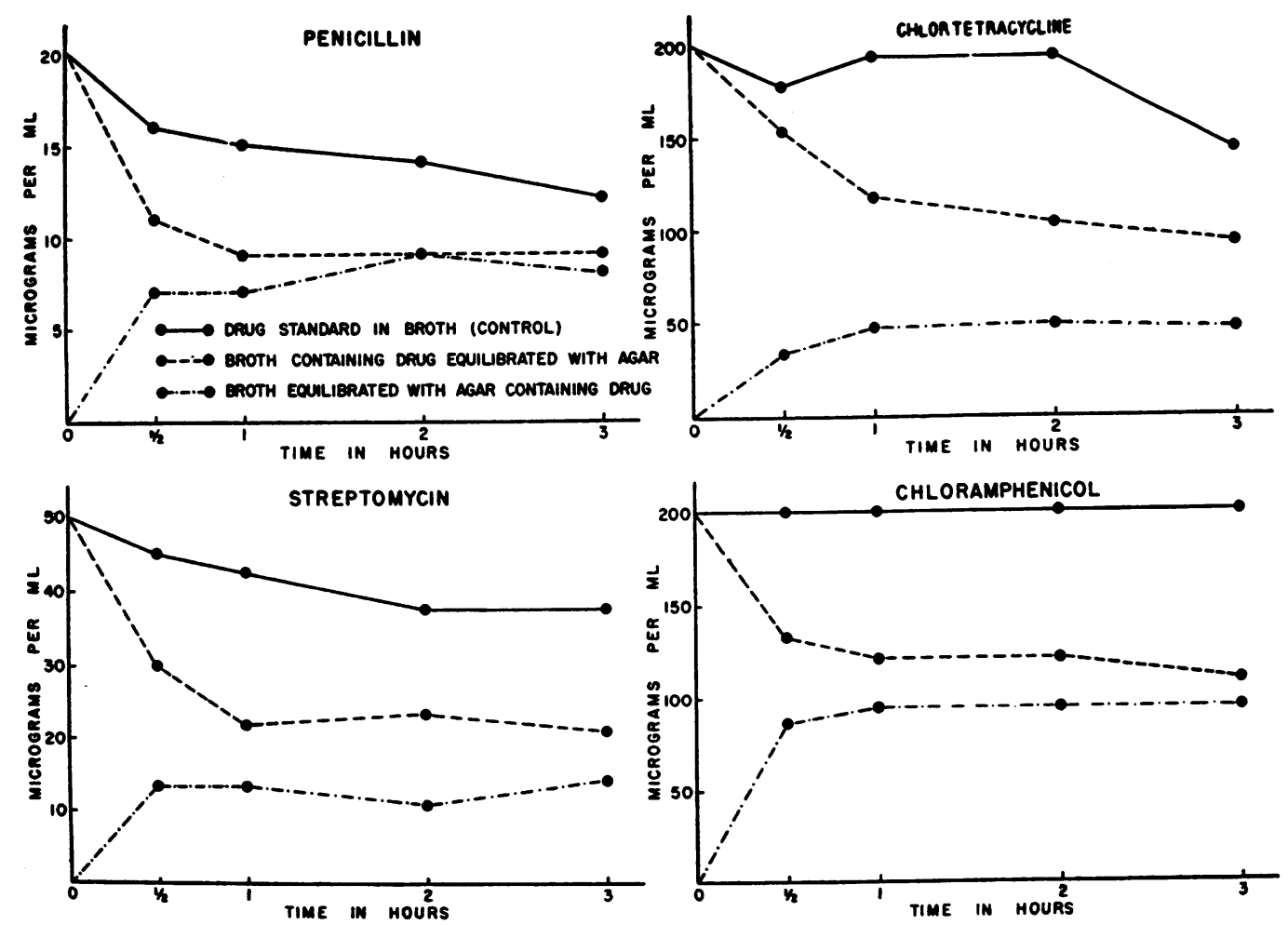

Fig. 7. Rate of Diffusion of Penicillin, Streptomycin, Chlortetracycline and Chloramphenicol between Broth and 3 Per Cent Agar In Vitro at $37^{\circ} \mathrm{C}$. 

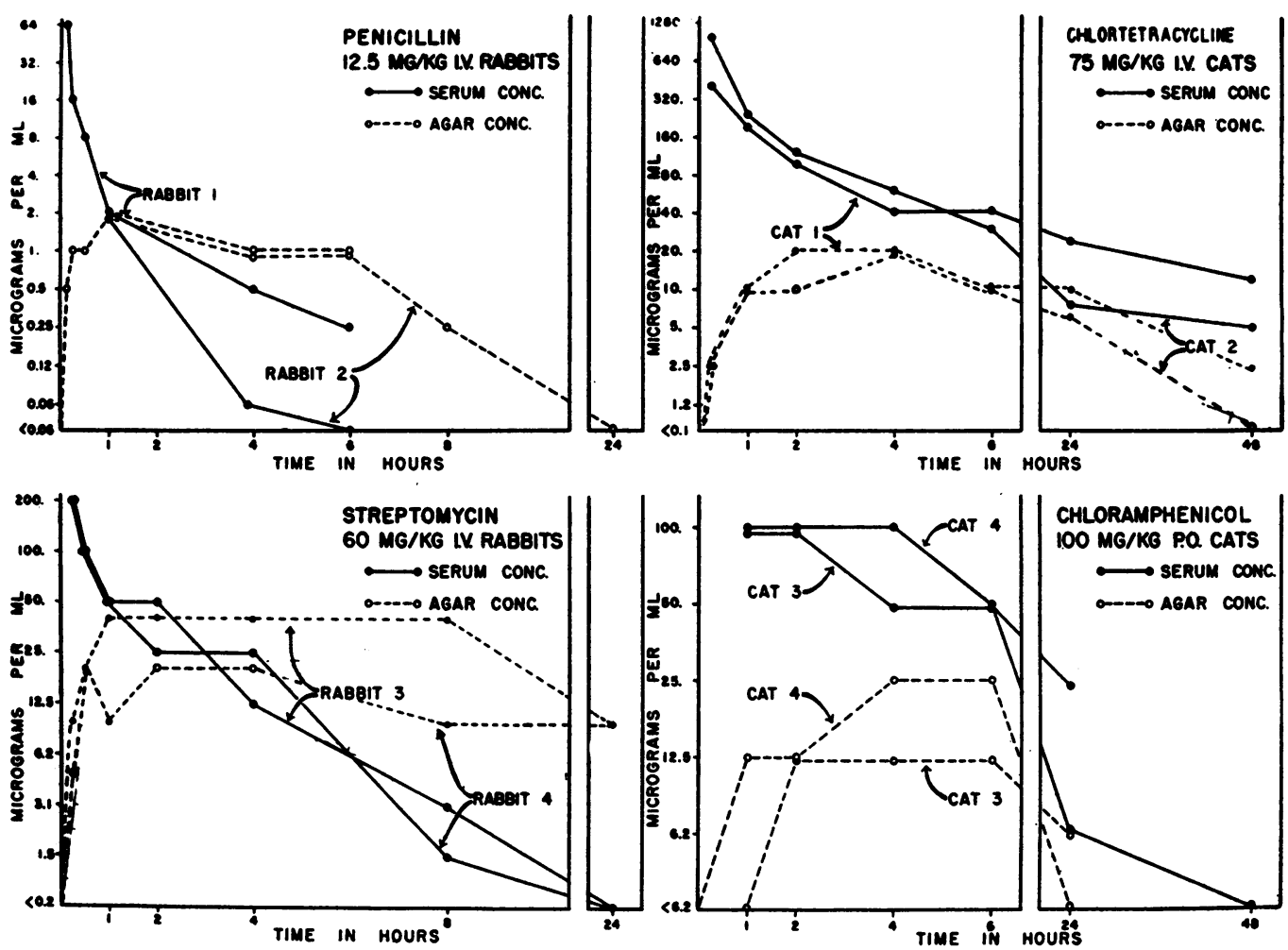

Fig. 8. Relation of Concentrations of Penicillin, Streptomycin, Chlortetracycline and Chloramphenicol in Intraperitoneal Agar Discs to Serum Concentrations

\section{DISCUSSION}

The above observations indicate that it is possible by means of the triple layered agar discs to localize a microbial population effectively within the experimental animal in such a manner that quantitative determinations of bacterial growth can be made at regular intervals. In this situation the microorganisms were found to be subsisting upon nutriments supplied by the extracellular fluid of the host in a state more closely approximating true parasitism than would be possible in vitro. While it is true that certain species of pathogenic bacteria such as $\mathrm{Kl}$. pneumoniae were able to grow to a limited degree on 3 per cent agar to which no nutriment was added, this growth was not comparable to the luxuriant growth of the bacteria which occurred in the agar discs implanted in the peritoneal cavity of the experimental animal.

The bacteria growing in the agar discs were effectively protected from attack by host macrophages and yet were exposed to the action of chemotherapeutic drugs which quickly passed through the host body fluids to penetrate the agar discs implanted in the peritoneal cavity.

The triple layered agar discs used in these experiments were easy to construct in the laboratory, and with practice a considerable number could be prepared in a short time. Thermal killing of the organisms did not occur as long as the temperature of the agar was carefully regulated. Three per cent unenriched agar constituted an adequate matrix for bacterial multiplication, and the transparency of the agar enabled colonies to be seen directly in the depths of the discs. The bacteria could be recovered for subculture and further study such as drug-susceptibility determinations after incubation in vivo. The rubbery consistency and the flat shape of the discs permitted easy handling, and discs remained intact in the peritoneal cavity for as long as three weeks and in broth in petri dishes for as long as four weeks. Only an occasional disc was lost in the peritoneal cavity due to crumbling, and a disc could usually be recovered whole even when it had slipped out of the encircling aluminum cylinder. 
One deficiency of the agar disc technic was that the enclosed organisms were not always completely confined and peritoneal contamination sometimes resulted. The incidence of contamination varied with: the care with which the discs were prepared and introduced into the animals; the number of discs inserted and the size of the bacterial inoculum; the time of incubation within the animals; and the organism which was selected. As proficiency in technic was acquired the frequency of peritoneal contamination diminished. In the few instances in which the peritoneal culture was found to be positive immediately after the discs containing bacteria had been inserted, it is not unlikely that contamination of the exterior of the discs may have occurred from aerosol created during the pouring of the middle layer. Nevertheless, the efficacy of agar in providing an effective medium for localizing large numbers of bacteria was demonstrated by the fact that discs containing 100 to 150 colonies of staphylococci or typhoid bacilli were removed from animals after 24 hours of incubation and the peritoneal cultures of these animals were sterile. Moreover, discs containing many colonies of bacteria remained in petri dishes flooded with broth for as long as 48 hours without contamination of the surrounding liquid medium.

The chief shortcoming of the agar discs in studying drug-host-parasite relationships in experimental animals is that they are an artificial and unphysiologic means of endeavoring to reproduce the conditions present during infection. The endresult produced by a drug on a microbial population in vivo involves the interaction of a number of factors which have to do with both drug-host and drug-parasite relationships (1). Nevertheless the present technic makes it possible to separate drug-bacteria reactions within a host from the host's own cellular reactions and may be helpful in defining the respective roles of cellular defense and drug effect in the study of a particular infection.

It is also believed that the foregoing experimental method will have a degree of usefulness in the study of other biologic phenomena pertaining to chemotherapy and the behavior of bacterial populations in animal hosts. By mixing bacteria in the middle layer of the disc with purulent exudate or caseum, for example, it may be possible to determine the degree to which these products of natural infection compromise drug action. The formation of a thick, fibrinocellular film about the agar discs in rabbits affords additional means of investigating the ability of antimicrobial drugs to penetrate such membranes. In the present study it is significant that penicillin given in large doses to rabbits more readily penetrated the exudate encasing agar discs in the peritoneal cavity than solid fibrin clots implanted subcutaneously as reported elsewhere (7).

Finally, the use of this technic promises to serve as a critical test of the ability of new chemotherapeutic substances to pass the several anatomicophysiologic barriers which exist in the host between the site of administration of a drug and its locus of antimicrobial action in the extracellular fluid. In this way the use of the agar discs may help to explain certain discrepancies of drug action in vivo and in vitro. Such an investigation into the differences in antityphoidal action of chlortetracycline and chloramphenicol is the subject of a separate report (8).

\section{SUMMARY}

A method has been outlined whereby a bacterial population has been successfully localized in the peritoneal cavity of an animal host for varying periods of time. The bacteria were localized in the central portion of triple layered 3 per cent agar discs and were completely isolated by surrounding transparent agar. As no nutriment was added to the agar, the bacteria were dependent for growth on nutritive substances from the surrounding tissue fluids and were able to exist in a state more closely approximating true parasitism than is possible in vitro. The tissue reaction to the agar discs in the peritoneal cavity resembled a foreign body reaction. Exudate containing inflammatory cells lined the surface of the discs, but the cells were not able to penetrate the substance of the agar.

Pathogenic bacteria were tested for their ability to grow in several animal species in this situation. Staphylococci, typhoid bacilli, Friedlander's bacilli, and anthrax bacilli grew luxuriantly in agar discs in rabbits, guinea pigs, and cats, although growth had been negligible in unenriched agar in vitro. Streptococci, enterococci, pneumococci, and brucella also grew well in the agar discs in vivo, although the colonies sometimes attained only micro- 
scopic size. Contamination of the peritoneal cavity occurred in less than 25 per cent of trials.

The agar discs were well penetrated in vitro and in vivo by penicillin, streptomycin, chlortetracycline, and chloramphenicol. It is expected that this method will be useful in assessing the direct effect of antimicrobial compounds in the extracellular fluids of animal hosts and in studying other biologic phenomena.

\section{REFERENCES}

1. McDermott, W., Host factors in chemotherapy, in Dubos, R. J., ed., Bacterial and Mycotic Infections of Man, ed. 2, Philadelphia, J. B. Lippincott, 1952, p. 744.

2. Eagle, H., Fleischman, R., and Musselman, A. D., The bactericidal action of penicillin in vivo: the participation of the host, and the slow recovery of the surviving organisms. Ann. Int. Med., 1950, 33, 544.

3. Lurie, M. B., Studies on the mechanism of immunity in tuberculosis. The fate of tubercle bacilli in- gested by mononuclear phagocytes derived from normal and immunized animals. J. Exper. Med., 1942, 75, 247.

4. Lurie, M. B., On the mechanism of immunity in tuberculosis. The host-parasite relationship under the conditions of a localized agar focus of infection and the generalization of the disease in normal and immunized rabbits. J. Exper. Med., 1936, 63, 923.

5. Frost, W. D., The antagonism exhibited by certain saprophytic bacteria against the bacillus typhosus gaffky. J. Infect. Dis., 1904, 1, 599.

6. Tompsett, R., Shultz, S., and McDermott, W., The relation of protein binding to the pharmacology and antibacterial activity of penicillins $\mathrm{X}, \mathrm{G}$, dihydro $\mathrm{F}$, and K. J. Bact., 1947, 53, 581.

7. Weinstein, L., Daikos, G. K., and Perrin, T. S., Studies on the relationship of tissue fluid and blood levels of penicillin. J. Lab. \& Clin. Med., 1951, 38, 712.

8. Werner, C. A., and McDermott, W., Studies of microbial populations artificially localized in vivo. II. Difference in antityphoidal activities of chloramphenicol and chlortetracycline. J. Clin. Invest., 1954, 33, 753. 